Watanabe, T. (1960). J. gen. Microbiol. 22, 102-112

\title{
Transductional Studies of Thiamine and Nicotinic Acid requiring Streptomycin Resistant Mutants of Salmonella typhimurium
}

\author{
Bx T. WATANABE* \\ Department of Zoology, Columbia University, New York City, New York, U.S.A., and \\ Department of Bacteriology, Keio University, School of Medicine, Tokyo, Japan
}

SUMMARY : By selection with concentrations of streptomycin from 25 to $250 \mu \mathrm{g} . / \mathrm{ml}$., auxotrophic mutants were obtained with high frequency from Salmonellatyphimurium strain LT-2. All of them were found to require both thiamine and nicotinic acid for their growth. The requirement for thiamine can be satisfled by either 4-methyl-5hydroxyethyl-thiazole, cystine or cysteine but not by methionine. Nicotinic acid can be replaced by nicotinamide. No other amino acid, vitamin, purine or pyrimidine can replace these vitamins.

In addition to the nutritional requirement, all of these mutants are streptomycin resistant slow growers. These four characters, i.e. thiamine and nicotinic acidrequirement, slow growth and streptomycin resistance are converted to wild-type in a single step by transduction with phage PLT-22, grown on wild-type bacteria as well as on prototrophic one-step intermediate streptomycin resistant mutants and streptomycin indifferent mutants. Spontaneous mutants with wild phenotype were also obtained from some unstable mutants of this group, apparently in a single mutational event. The transductional findings suggest that these auxotrophic mutants are probably due not to some chromosomal aberration but to mutation within a single locus. The data from reciprocal transductions between these mutants suggest that multiple sites of the same locus are responsible. The mutations at different sites seem to have different levels of mutability and some of them have different levels of streptomycin resistance.

In the course of transduction studies of streptomycin resistance and sensitivity in Salmonella typhimurium we found that several one-step intermediate resistant mutants were auxotrophs (Watanabe \& Watanabe, 1959b). Although they were isolated from several independent cultures, all of them were found to require both thiamine and nicotinic acid for their growth and to be invariably slow growers. At first this group of mutants was thought to arise from some chromosomal aberration, but recent studies have disclosed that they are due to mutations within a single locus.

As pointed out in a previous paper (Watanabe \& Watanabe, 1959b), the mode of action of streptomycin and the biochemical mechanism of streptomycin resistance in bacteria are not well known. If the streptomycin resistance of these mutants is brought about by a biochemical block which leads to thiamine and nicotinic acid-requirement, this might offer an important clue to studying these problems. On the other hand, nothing is known about the metabolic interrelationship between thiamine and nicotinic acid, and these

* Present address: Department of Bacteriology, Keio University, School of Medicine, Tokyo, Japan. 
mutants are the first ones which suggest the presence of some metabolic interrelationship between them. These mutants seem to be of particular interest and importance in these respects and genetic and biochemical studies of them will be reported in the present paper.

\section{METHODS}

\section{Strains and media}

Salmonella typhimurium strain LT-2 and a temperate phage PLT-22 were exclusively used as our transduction system. This bacterial strain has rather high resistance to streptomycin as previously mentioned (Watanabe \& Watanabe, $1959 a$ ), and in order to make the analysis of streptomycin resistance easier the low phosphate medium (Watanabe \& Watanabe, 1959a) was again used in the present studies. Except when streptomycin resistance was to be determined, the following high phosphate minimal medium was used, either with or without enrichment with yeast extract and NZ-case (in \%): $\mathbf{N H}_{4} \mathrm{Cl}$, $0.5 ; \mathrm{NH}_{4} \mathrm{NO}_{3}, 0.1 ; \mathrm{Na}_{2} \mathrm{SO}_{4}, 0.2 ; \mathrm{K}_{2} \mathrm{HPO}_{4}, 0.8 ; \mathrm{KH}_{2} \mathrm{PO}_{4}, 0.1 ; \mathrm{MgSO}_{4}, 0.01$; $\mathrm{CaCl}_{2}, 0.001$; yeast extract (DIFCO), 0.5; NZ-case (Sheffield), 0.5; (agar, 1.5). In these low phosphate and high phosphate media, the growth attained by wild-type bacteria did not differ considerably from each other, especially when they were enriched with yeast extract and NZ-case.

\section{Isolation and characterization of mutants}

Streptomycin resistance has been classified by us (Watanabe, 1954; Watanabe, Fukasawa \& Ushiba, 1957; Watanabe \& Watanabe, 1959a) into streptomycin indifference (one-step complete resistance) and multiple-step resistance. The mutation rate to streptomycin indifference in Salmonella typhimurium strain LT-2 is very low (approximately $10^{-10}$ per bacterium per division cycle), and studies of its transduction have been reported previously (Watanabe \& Watanabe, 1959a).

The streptomycin resistance which we are going to deal with here is onestep intermediate resistance. The way to isolate one-step intermediate resistant mutants was described elsewhere (Watanabe \& Watanabe, 1959a). From each of 15 independent overnight cultures of wild-type bacteria in enriched broth started from about 100 cells, $0.1 \mathrm{ml}$. was spread on plates of the low phosphate agar medium containing $25,50,100$ and $250 \mu \mathrm{g}$. of streptomycin sulphate per $\mathrm{ml}$. Three of the colonies which developed on each plate after 5 days' incubation at $37^{\circ}$ were picked at random and purified by repeating single colony isolations on antibiotic-free enriched agar plates. They were finally streaked on both enriched and minimal agar to see whether or not they were auxotrophs. Those which were found to be auxotrophs were then studied for their nutritional requirement with the auxanographic technique (Lederberg, 1950). Isolation of thiamine and nicotinic acid-requiring mutants by the penicillin screening method (Davis, 1949) was also attempted. Overnight cultures of wild-type bacteria in enriched broth were washed three times with 
saline and finally resuspended in the original amount of saline. One-tenth $\mathrm{ml}$. of undiluted and 10-1-diluted suspensions was inoculated into $3 \mathrm{ml}$. of minimal broth containing 50 units penicillin $\mathrm{G} / \mathrm{ml}$. The inoculated media were incubated at $37^{\circ}$ for $24 \mathrm{hr}$. without aeration. One-tenth ml. of each suspension was then plated on minimal agar containing both thiamine hydrochloride and nicotinic acid ( $2 \mu \mathrm{g} . / \mathrm{ml}$.). This concentration of each vitamin was found to be sufficient to support the growth of thiamine and nicotinic acid-requiring mutants and not to be inhibitory to them. Colonies which developed on the minimal agar containing thiamine and nicotinic acid were picked and streaked on both minimal and enriched agar plates to characterize auxotrophs.

\section{Mutability to wild-type of streptomycin resistant mutants}

Mutability was tested by transferring the mutant cultures in enriched broth or on enriched agar for many generations to see whether or not any fast-growing reversions developed spontaneously. A large number of cells of one mutant strain, which proved highly stable, was inoculated into minimal broth and agar, after being thoroughly washed with saline, to see if any prototrophic reversions appeared. Cultures of this stable, auxotrophic strain were also subjected to ultraviolet irradiation and $\mathrm{MnCl}_{2}$ treatment (Demerec \& Flint, 1951), both fast growing and prototrophic mutants being looked for. The mutagen-treated cells were incubated at $20^{\circ}, 27^{\circ}$ and $37^{\circ}$ as a check on possible temperature effects.

\section{Transduction experiments}

The preparation of phage stocks was described in a previous paper (Watanabe \& Watanabe, $1959 a$ ). When auxotrophic mutants were used as recipients in transduction, they were plated, after infection by phage in enriched liquid medium, on minimal agar enriched with either thiamine, nicotinic acid, thiamine plus nicotinic acid or yeast extract plus NZ-case in some experiments, while in others the phage-infected bacteria were thoroughly washed free of nutrients and then plated on minimal agar. The inoculated plates were incubated at $20^{\circ}, 27^{\circ}$ and $37^{\circ}$.

Transductant colonies were examined for fast growth, streptomycin sensitivity and prototrophy by determining their growth rates by turbidimetry with a Klett-Summerson photoelectric colorimeter, by streaking on minimal agar and also by determining their survival curves on streptomycin agar.

Linkage studies were made, by the transduetion technique, between this group of mutants and other Salmonella typhimurium auxotrophic strains having different nutritional deficiencies. The mutants of this group were infected with phage grown on the auxotrophic strains and then plated on enriched agar; the large colonies which developed were replica plated to appropriate media for scoring their characters (Lederberg \& Lederberg, 1952). Thus when streptomycin indifferent mutants were used as donors, the transductants were replicated on to enriched agar containing $500 \mu \mathrm{g}$. of streptomycin per ml. When auxotrophic mutants were used as donors, the trans- 
ductant colonies were replicated on minimal agar to see if some of them were auxotrophs.

In the transduction of streptomycin sensitivity, fast growth and prototrophy into the present group of mutants, the presence of abortive transductants was looked for by the method of Ozeki (1956; see also Watanabe \& Watanabe, $1959 a, b)$

\section{RESULTS}

As described above, $0.1 \mathrm{ml}$. of each of 15 independent broth cultures of wild type Salmonella typhimurium strain LT-2 were plated on low phosphate agar containing 25, 50, 100 and $250 \mu \mathrm{g} . / \mathrm{ml}$. streptomycin. A total of 138 colonies developed on the 25,50 and $100 \mu \mathrm{g} . / \mathrm{ml}$. streptomycin plates. When these colonies were streaked on both minimal agar and enriched agar, 15 were found to be auxotrophs. These 15 auxotrophic mutant strains originated from 9 of the 15 initial cultures. Three of the initial cultures yielded three auxotrophic mutant colonies each. A total of 11 colonies developed on $250 \mu \mathrm{g} . / \mathrm{ml}$. but none of them was found to be auxotrophic and none of the 15 cultures gave colonies on the low phosphate agar medium containing more than $500 \mu \mathrm{g} . / \mathrm{ml}$. of streptomycin.

\section{Phenotypes of one-step intermediate streptomycin-resistant auxotrophic mutants}

Streptomycin resistance. Average streptomycin resistance was determined by plating several hundred cells of each mutant on agar containing varying concentrations of streptomycin. The maximal concentration on which the mutant gave colony counts approximately equal to that on the control plates without streptomycin was called its average resistance. All of the mutant strains except one had their average resistance between 100 and $250 \mu \mathrm{g} . / \mathrm{ml}$. and the exceptional one had its average resistance between 50 and $100 \mu \mathrm{g} . / \mathrm{ml}$. These results were reproducible.

Mutability of the mutants. It was found that 4 out of 9 independently isolated mutants were genetically quite unstable and easily reverted to wild phenotype, apparently in a single spontaneous step, whereas the other mutants were rather stable. Fast-growing reversions arising on complete medium were all streptomycin sensitive prototrophs. Prototrophic reversions selected on minimal medium were also all fast growing and streptomycin sensitive. The mutability of the auxotrophic mutants was not studied quantitatively, but some of them were apparently more mutable than the others. One of the mutants, which was named no. $1 a$, was quite stable and refractory to both ultraviolet and $\mathbf{M n C l}_{2}$. Mutants named no. $1 b$ and no. $1 c$ which were originally isolated from the same culture as no. $1 a$ were also quite stable. A total of about $10^{13}$ washed cells of mutant no. $1 a$ were inoculated, in several experiments, into minimal medium but no prototrophic mutants could be recovered. About $10^{10}$ cells of this mutant were treated with either u.v. light or $\mathrm{MnCl}_{2}$ under various conditions, but the mutant cells were quite stable, giving rise to no prototrophic mutants. The different temperatures at which 
the treated mutant cells were incubated had no effects on the production of induced revertants in this mutant.

Nutritional requirement of the mutants. All of the mutants were found to require both thiamine and nicotinic acid for their growth. Cystine, cysteine and 4-amino-5-hydroxyethyl-thiazole were found to be able to replace thiamine. No other vitamin, amino acid or purine and pyrimidine, nor mixtures of these, were able to support growth. Yura (1956) reported on adenine-thiamine mutants of Salmonella typhimurium, but growth of our mutants was not supported by the mixture of thiamine and adenine. Although this salmonella strain and its mutants were found to be inhibited by high concentrations of adenine, no growth occurred when adenine and thiamine were separately spotted a short distance apart on minimal agar seeded with the auxotrophic mutants.

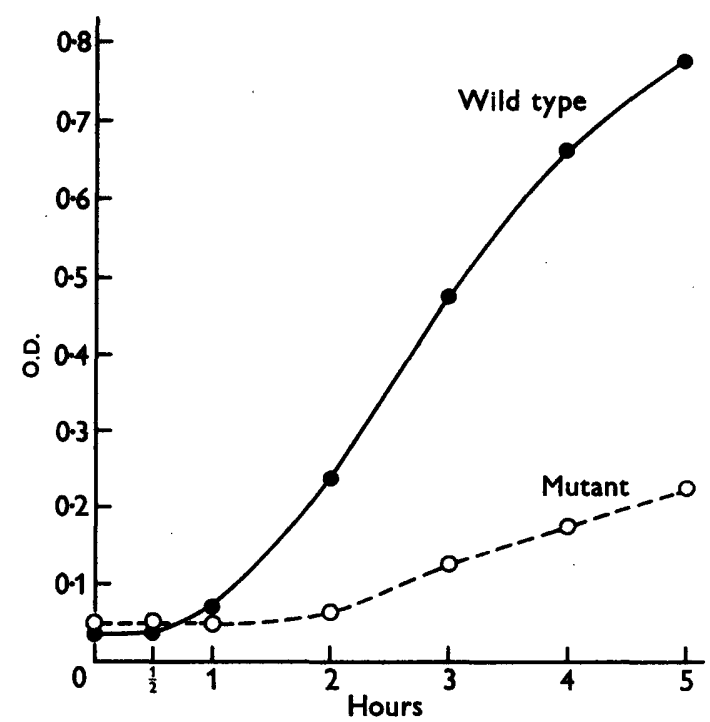

Fig. 1. Growth curves of wild-type and a thiamine and nicotinic acid-requiring streptomycin-resistant mutant of Salmonella typhimurium strain LT-2 in minimal medium enriched with yeast extract and NZ-case (0.5\% respectively).

Nicotinic acid was shown to be synthesized by way of tryptophan in mammals (Dalgliesh, 1951) and Neurospora (Bonner \& Yanofsky, 1951), but not in Escherichia coli and Bacillus subtilis (Yanofsky, 1954). It is not known what the precursors of nicotinic acid are in these bacteria. In the present mutants nicotinamide was able to replace nicotinic acid, but tryptophan and the intermediates between tryptophan and nicotinic acid, i.e. kynurenine, oxy-kynurenine and 3-oxy-anthranilic acid (quinolinic acid was not available) could not, even in the presence of thiamine.

Thiamine is considered to be synthesized by the condensation of 2-methyl4-amino-5-hydroxymethyl-pyrimidine and 4-amino-5-hydroxyethyl-thiazole (Tatum \& Bell, 1946) and the latter compound is assumed to be synthesized 


\section{Transductional studies with Salmonella typhimurium 107}

by the condensation of methionine, acetaldehyde and ammonia (Bonner, 1987). In this regard, it is interesting to note that methionine could not replace thiamine in these mutants, whereas cystine and cysteine could.

Growth curves of the mutants showed that high concentrations of nicotinic acid (more than $20 \mu \mathrm{g} . / \mathrm{ml}$.) were inhibitory, but thiamine was not.

Slow growth. As seen in Fig. 1, the mutants gave very long generation times in the enriched medium which could not be shortened by the addition of more thiamine and nicotinic acid to the medium.

Effects of thiamine and nicotinic acid on the streptomycin resistance of the mutants. When the survival curves of the mutants were determined on both minimal agar and enriched agar with either low phosphate or high phosphate concentrations and with varying concentrations of thiamine and nicotinic acid, it was found that as long as these vitamins were contained in the media in concentrations sufficient to support growth, the survival curves were identical. In the absence of these vitamins it was naturally impossible to determine their streptomycin resistance.

\section{Transductional analysis of the mutants}

Transduction with phage grown on wild type bacteria. As reported previously (Watanabe \& Watanabe, 1959b), infection of mutants of this group with phage grown on wild-type bacteria produced wild-type transductants. The results are shown in Table 1. The large colonies on complete agar and the colonies on minimal agar were re-isolated and tested for their growth rates,

Table 1. Transduction of roild-type into a thiamine and nicotinic acid-requiring streptomycin-resistant mutant of Salmonella typhimurium strain LT-2 with phage PLT-22 grown on wild-type bacteria

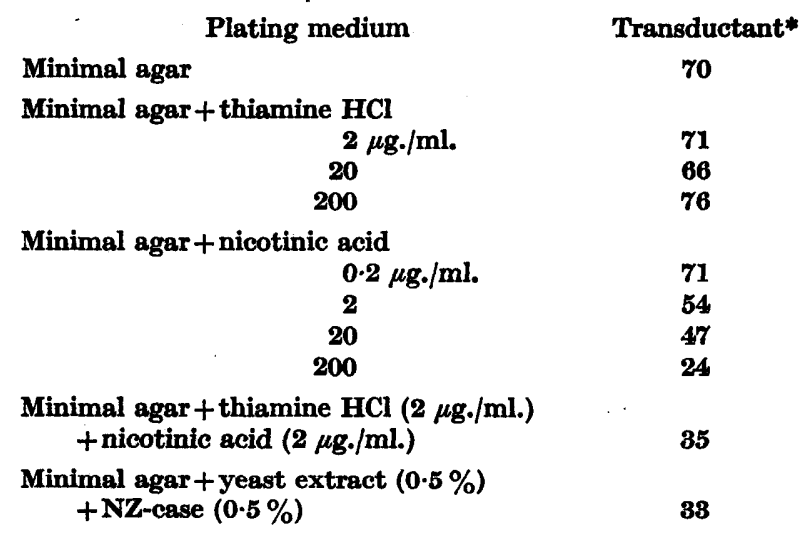

Phage-infected bacteria were thoroughly washed with saline before plating. Equal volumes of phage $\left(10^{\circ} / \mathrm{ml}\right.$.) and bacteria (about $10^{\circ} / \mathrm{ml}$.) were mixed and $0 \cdot 1 \mathrm{ml}$. of the mixture was plated on each plate.

* All of the transductant colonies (colonies developed on minimal agar and partially enriched minimal agar and large colonies developed on complete medium) were found to be fast growing, streptomycin-sensitive prototrophs. Transductant count is the total of those developed on five plates. 
nutritional requirement and streptomycin-sensitivity. It was found that these colonies were invariably streptomycin sensitive prototrophs which had equal growth rate to wild-type bacteria. It will be seen from these data that all of these characters, i.e. slow growth, streptomycin resistance and thiamine and nicotinic acid-requirement were converted to wild-type in a single step by transduction and that there was no segregation among these characters. Another fact revealed by this table is that the number of transductant colonies developed on the medium enriched with either yeast extract pluz NZ-case or thiamine plus nicotinic acid is slightly less than those found on the other plates. This tendency was consistently observed in repeated experiments not shown here. This finding suggested that the background growth of nontransductants possibly suppresses the formation of transductant colonies. Reconstruction experiments, however, failed to confirm this.

Transduction with phage grown on one-step intermediate streptomycin resistant prototrophic mutants. It was previously reported (Watanabe \& Watanabe, $1959 b$ ) that multiple loci which are not linked to the streptomycin indifference locus were found by transduction. At least seven different loci which control one-step intermediate streptomycin resistance were found (unpublished data). As in the case of prototrophic one-step intermediate streptomycin resistant mutants, infection of the present series of mutants with the phage grown on these prototrophic resistant mutants was found to give wild-type transductants with the frequencies about equal to the phage from wild-type bacteria. The transductants obtained here were again found to be all prototrophic, fast growing and streptomycin sensitive.

Transduction with phage grown on streptomycin indifferent mutants. The transduction of streptomycin indifference into sensitive bacteria and also into one-step intermediate resistant prototrophic mutants was reported previously (Watanabe \& Watanabe, 1959a); it was also reported that phage grown on indifferent mutants could transduce streptomycin sensitivity and fast growth into one-step intermediate resistant, slow growing (but prototrophic) mutants as revealed by selecting large colonies on the poor background growth of non-transductants. Similar results were obtained with the present series of auxotrophic mutants. In other words, streptomycin indifference was transducible into these mutants with the frequency of about $5 \times 10^{-5}$ per phage and the streptomycin indifferent transductants were still slow growers which required both thiamine and nicotinic acid for their growth. When fast-growing transductants or prototrophic transductants were looked for, they were found to be wild-type also in other characters. Accordingly, no linkage was found between the streptomycin indifference locus and that which controls streptomycin resistance, slow growth and thiamine and nicotinic acid-requirement.

Attempts to detect linkages of the mutant locus with other loci determining auxotrophy. Eleven auxotrophic mutants of strain LT-2 were employed which had been found to belong to different linkage groups in transduction by Demerec and his group. They were pro-13, pro-22, leu-A-3, leu-B-28, ser-5, cys-7, cys-20, meth-6, try-D-10, his-8 and gly-1. When the present 
series of thiamine and nicotinic acid-requiring mutants were infected with the phage grown on each of these auxotrophs, fast growing streptomycin-sensitive transductants developed. All of the transductant colonies, however, were found to be prototrophs and no linkage relationship was detected by this transduction technique between the locus characterizing the present mutants and the auxotrophic loci tested.

Reciprocal transductions among the mutants. Some of the mutants were so mutable that they could not be used for our transduction studies. The results of the reciprocal transductions are shown in Table 2. As seen from this table, the mutants of this group gave small numbers of wild-type transductants, whereas they gave frequent wild-type transductants with the phage grown on wild-type bacteria. The crosses between the mutants which were isolated from the same culture did not give any wild-type transductants, suggesting that they were of clonal origin.

Table 2. Reciprocal transduction to wild type among thiamine and nicotinic acid-requiring streptomycin-resistant mutants of Salmonella typhimurium strain LT-2 with phage PLT-22

\begin{tabular}{|c|c|c|c|c|c|c|c|c|}
\hline \multirow[b]{2}{*}{ Donor } & \multicolumn{8}{|c|}{ Recipient } \\
\hline & No. $1 a$ & No. $2 a$ & No. $3 a$ & No. 4 & No. 5 & No. $1 b$ & No. $2 b$ & No. $3 b$ \\
\hline No. $1 a$ & o & 7 & 2 & 2 & $\mathbf{1}$ & $\mathbf{0}$ & - & - \\
\hline No. $2 a$ & 14 & o & 41 & 51 & 41 & - & $\mathbf{0}$ & - \\
\hline No. $\mathbf{3} a$ & $\mathbf{3}$ & 43 & 0 & 11 & 4 & - & - & o \\
\hline No. 4 & 4 & 39 & 10 & $\mathbf{0}$ & 3 & - & - & - \\
\hline No. 5 & 0 & 30 & 5 & 13 & 0 & - & - & - \\
\hline No. $1 b$ & $\mathbf{0}$ & - & - & - & - & $\mathbf{0}$ & - & - \\
\hline No. $2 b$ & 一 & $\mathbf{0}$ & - & - & - & - & $\mathbf{0}$ & - \\
\hline No. $3 b$ & - & - & o & - & - & - & - & o \\
\hline Wild-type & 186 & 204 & 270 & 287 & 248 & - & - & - \\
\hline No phage & 0 & 0 & 0 & 0 & 0 & 0 & o & o \\
\hline
\end{tabular}

The figures represent the transductant counts on each minimal agar plate. Equal volumes of phage $\left(10^{10} / \mathrm{ml}\right.$.) and bacteria (about $10^{\circ} / \mathrm{ml}$.) were mixed and $0 \cdot 1 \mathrm{ml}$. of the mixture was plated on each plate.

No. $1 a$ and no. $1 b$, no. $2 a$ and no. $2 b$ and no. $3 a$ and no. $3 b$ came from the same cultures respectively.

Abortive transduction. When the mutants were infected with phage from wild-type bacteria and plated on minimal agar and minimal agar enriched to varying extent with either yeast extract plus NZ-case, thiamine, nicotinic acid or the mixture of these vitamins, large transductant colonies developed on both the enriched medium and the deficient media, as mentioned above. The presence of smaller colonies, indicating abortive transductions, was carefully looked for with a microscope but not found. It was suspected, therefore, that some of the large colonies might have been abortive transductants which could somehow grow fast. More than 200 colonies were reisolated on minimal agar by Ozeki's method (1956), but all of them gave massive growth of prototrophic colonies. Abortive transductions were looked for also by incubating the original plates inoculated with the phage-infected 
recipient bacteria, not only at $37^{\circ}$ but also at $27^{\circ}$ and $20^{\circ}$ respectively, but no abortive transductants were found at any of these temperatures. The reason of this failure is not known.

Transduction of streptomycin resistance into wild-type bacteria with phage grown on the present series of mutants. Streptomycin resistance was again not transduced into wild-type bacteria in repeated experiments in which the phage-infected bacteria were allowed to grow in the enriched liquid medium or agar medium for varying durations after infection in order to allow them to express their phenotype before the selective concentration of streptomycin was added. The same failure was experienced before (Watanabe \& Watanabe, 1959 a) with prototrophic one-step intermediate streptomycin-resistant mutants. The reason of this failure is again unknown.

\section{DISCUSSION}

Among one-step intermediate streptomycin resistant mutants which were isolated from Salmonella typhimurium strain LT-2 by selecting with intermediate concentrations of streptomycin on a complete medium, mutants which require both thiamine and nicotinic acid for their growth were found with a high frequency. About $11 \%$ of the total mutants tested were auxotrophs which were all found to belong to this type and 9 out of 15 independent cultures gave at least one colony of this mutant type, when 9 colonies were tested from each culture. It would not be unreasonable to assume that the mutants of this type could have been detected from more cultures, if more colonies were picked and tested. When it was attempted to isolate mutants of this type with the penicillin screening method, however, no mutants were isolated in three independent experiments. It might mean that this type of mutation is induced by streptomycin as Sevag \& Rosanoff (1952) concluded on their phenylalanineless mutants of Staphylococcus aureus. The irregular distribution of the mutants among the independent cultures, on the other hand, may suggest that they are spontaneous mutants and that they are selected by streptomycin because they are resistant to this antibiotic. This latter view seems more likely, because the mutants from the same culture were found to be genetically homogeneous by transduction, while those from different cultures were found to be genetically heterogeneous. On account of the failure to isolate the mutants of this type with the penicillin screening method, it was not possible to isolate thiamine and nicotinic acid-requiring mutants without exposing the bacteria to streptomycin and selecting for streptomycin resistance. The reason for the failure is possibly because the mutants could grow in minimal medium containing penicillin by utilizing trace amounts of vitamins and were killed by penicillin.

From the transduction data that streptomycin resistance, slow growth and thiamine and nicotinic acid-requirement were converted to streptomycin sensitivity, fast growth and prototrophy in a single step, by means of phage grown on wild-type bacteria, and that these characters did not segregate from each other, it is clear that they are controlled by a single locus. They are not 


\section{Transductional studies with Salmonella typhimurium}

brought about by some chromosomal aberration in view of the fact that reciprocal transductions were possible among the mutants of this group and the frequencies of reciprocal transductions were very low suggesting that they are due to mutations at different mutational sites of the same locus, although this is not quite certain yet, since no other markers linked to this mutant marker have been discovered. Their average resistance to streptomycin was determined and it was found that one of the mutants had definitely lower resistance than the others. Mutability also differed considerably from mutant to mutant.

Although the remarkable biochemical feature of these mutants has not been elucidated, it is obvious that there is a close interrelationship between thiamine and nicotinic acid from our preliminary studies on the biochemistry of these mutants, because these requirements were found to be due to a single mutational event. In these mutants nicotinic acid could be replaced by nicotinamide but not by tryptophan nor the intermediates between tryptophan and nicotinic acid. Therefore, the biochemical block in these mutants is probably not located on the tryptophan-nicotinic acid pathway but rather it may suggest the absence of this pathway as in Escherichia coli and Bacillus subtilis (Yanofsky, 1954) and the presence of some unknown pathway related to both nicotinic acid and thiamine. The possibility that the block lies between 3-oxy-anthranilic acid and nicotinic acid cannot be excluded, because quinolinic acid was not available. Even if this compound were available, the block between it and nicotinic acid could not be excluded. The fact that thiamine was replaceable by cystine and cysteine is also difficult to understand, because methionine, which is supposed to be a precursor of thiamine (Bonner, 1937), could not replace this vitamin.

Another important feature of these mutants is that streptomycin resistance and slow growth are also brought about by the same locus. One may suspect that the slow growth is caused by the biochemical block which leads to the requirement of thiamine and nicotinic acid and that streptomycin resistance is the direct result of slow growth. Mere slow growth, however, does not usually give streptomycin resistance, and the streptomycin resistance of these mutants has to be considered to be due to some specific biochemical mechanism. Since it is produced by the same locus as the nutrition requirement, it is most reasonably assumed that the biochemical block somehow makes the cells resistant to streptomycin. Until the nature of the biochemical block in these mutants is elucidated, the mechanism of their streptomycin resistance remains a mystery. These mutants, however, offer a tool for studying both the mechanism of streptomycin resistance and the mode of action of streptomycin. On the other hand, the fact that the streptomycin resistance of these mutants is not changed by the addition of the required vitamins to the media and that most of the streptomycin-resistant mutants are prototrophs should be kept in mind. Our previous finding that there are multiple loci which control streptomycin resistance may indicate that there may be multiple mechanisms of streptomycin resistance among the different mutants. 
I am grateful to Dr Francis J. Ryan and Dr Daizo Ushiba for their encouragement in the present work. My thanks are also due to Dr M. Demerec for supplying his strains, to Dr Norihiko Hasegawa for the precursors of nicotinic acid and to Dr Ryoji Hayashi for the precursors of thiamine.

This work was supported in part by research grants from the U.S. Public Health Service, the National Science Foundation and the American Cancer Society.

\section{REFERENCES}

Bonner, D. M. \& Yanofsky, C. (1951). The biosynthesis of tryptophan and niacin and their relationships. J. Nutr. 44, 603.

BonNer, J. (1937). Vitamin $B_{1}$ growth factor for higher plants. Science, 85, 183.

Dalglinsh, C. E. (1951). Biological degradation of tryptophan. Quart. Rev. chem. Soc., Lond. 5, 227.

Davrs, B. D. (1949). The isolation of biochemically deficient mutants of bacteria by means of penicillin. Proc. nat. Acad. Sci., Wash. 35, 1.

Demerec, M. \& Flint, J. (1951). Mutagenic action of manganous chloride. Cold Spr. Harb. Symp. quant. Biol. 16, 215.

LEDERBERG, J. (1950). Isolation and characterization of biochemical mutants of bacteria. Meth. med. Res. 3, 5.

LEDERBERG, J. \& LEDERBERG, E. M. (1952). Replica plating and indirect selection of bacterial mutants. J, Bact. 63, 399 .

OzEKr, H. (1956). Abortive transduction in purine-requiring mutants of Salmonella typhimurium. Genetic studies with bacteria. Publ. Carneg. Instn, no. 612, 97.

SEvaG, M. G. \& Rosanoff, E. I. (1952). Mechanism of the development of resistance to streptomycin. I. Origin of resistant strains. J. Bact. 63, 243.

TAtUM, E. L. \& BeLL, T. T. (1946). Neurospora. III. Biosynthesis of thiamine. Amer. J. Bot. 33, 15.

WATANABe, T. (1954). Genetic studies on the mechanism of acquired streptomycin resistance in microorganisms. Keio J. Med. 3, 193.

Watanabe, T., Furasawa, T. \& Ushiba, D. (1957). Probable absence of direct induction of bacterial resistance to streptomycin. J. Bact. 73, 770.

Watanabe, T. \& Watanabe, M. (1959 $a)$. Transduction of streptomycin resistance in Salmonella typhimurium. J. gen. Microbiol. 21, 16.

Watanabe, T. \& Watanabe, M. (1959b). Transduction of streptomycin sensitivity into resistant mutants of Salmonella typhimurium. J. gen. Microbiol. 21, 30.

YANOFSKY, C. (1954). The absence of a tryptophan-niacin relationship in Escherichia coli and Bacillus subtilis. J. Bact. 68, 577.

YURA, T. (1956). Suppressor mutations in purine-requiring mutants of Salmonella typhimurium. Genetic studies with bacteria. Publ. Carneg. Instn, no. 612, 68. 